\title{
Conhecimento de enfermeiros de um pronto atendimento sobre lesão por pressão: potencialidades e fragilidades vivenciadas
}

\author{
Knowledge of nurses from an emergency room about pressure ulcers: strengths and \\ weaknesses experienced
}
Conocimiento de los enfermeros de una unidad de atención de emergencias: potencialidades y debilidades experimentadas

Gabrieli Santos dos Santos Panerai ${ }^{\star *}$, Karine de Freitas Cáceres Machado ${ }^{1}$ Silomar Ilha ${ }^{1}$, Adriana Dall Asta Pereira ${ }^{1}$.

\begin{abstract}
RESUMO
Objetivo: Verificar o conhecimento dos enfermeiros atuantes em um pronto atendimento sobre lesão por pressão. Métodos: Estudo exploratório, descritivo, qualitativo, realizado com 12 enfermeiros de uma Unidade de Pronto Atendimento Municipal no Rio Grande do Sul. Os dados coletados em novembro de 2020, por meio de um questionário contendo questões mistas, norteadas pela técnica vinheta, foram submetidos a análise de conteúdo. Resultados: Dentre da equipe multidisciplinar de saúde, os enfermeiros são profissionais fundamentais para realizar o cuidado de pacientes com lesão por pressão, possuem conhecimentos sobre os fatores que predispõe e realizam um plano de cuidados necessários. Contudo, referem não se sentirem capacitados para este cuidado e, algumas vezes, há falta de material para esta demanda. Percebem, também, a necessidade de realizar capacitações para atualização e como a presença ou ausência da mesma impacta diretamente nas tomadas de decisão sobre os cuidados com o paciente. Conclusão: Os profissionais possuem conhecimentos sobre os fatores que predispõe a lesão por pressão e realizam plano de cuidados; observou-se também que não se sentem capacitados e que acham pertinente a educação permanente sobre a temática em questão.
\end{abstract}

Palavras-chave: Enfermagem, Lesão por pressão, Emergências, Pessoal de saúde.

\begin{abstract}
Objective: To check the knowledge of nurses working in an emergency room about pressure ulcers. Method: Exploratory, descriptive and qualitative study conducted with 12 nurses from a Municipal Emergency Care Unit in Rio Grande do Sul. The data collected in November 2020, through a questionnaire containing mixed questions, guided by the vignette technique, were submitted to content analysis. Results: Among the multidisciplinary health team, nurses are fundamental professionals to provide care for patients with Pressure Ulcer, they have knowledge about the factors that predispose and carry out a necessary care plan. However, they say they do not feel qualified for this care and, sometimes, there is a lack of material for this demand. They also perceive the need to conduct training to update and how the presence or absence of it directly impacts decision-making about patient care. Conclusion: That the professionals have knowledge about the factors predisposing to pressure ulcers and carry out a care plan; it was also noted that they do not feel trained and that they consider continuing education on the theme in question.
\end{abstract}

Keywords: Nursing, Pressure ulcer, Emergencies, Health personnel.

\section{RESUMEN}

Objetivo: Chequear el conocimiento de los enfermeros actuantes en una unidad de atención de emergencias sobre las úlceras por presión. Métodos: Estudio exploratorio, descriptivo, cualitativo, realizado con 12 enfermeros de una Unidad Municipal de Atención de Emergencias en Rio Grande do Sul. Los datos recolectados en noviembre de 2020, a través de un cuestionario con preguntas mixtas, guiadas por la técnica de viñeta, se sometieron al análisis de contenido. Resultados: Dentro del equipo multidisciplinario de salud,

${ }^{1}$ Universidade Franciscana (UFN), Santa Maria - RS. *E-mail: enf.gabrielisantos@gmail.com 
las enfermeras son profesionales fundamentales para brindar atención a los pacientes con lesiones por presión, tienen conocimiento sobre los factores que predisponen y realizan un plan de atención necesario. Sin embargo, dicen no sentirse capacitados para esta atención y, en ocasiones, falta material para esta demanda. También perciben la necesidad de realizar capacitaciones para actualizar y cómo su presencia o ausencia impacta directamente en la toma de decisiones sobre la atención al paciente. Conclusión: los profesionales tienen conocimiento sobre los factores que predisponen a las úlceras por presión y desarrollan un plan de cuidados; también se observó que no se sienten entrenados y que consideran la educación continua sobre el tema en cuestión.

Palabras clave: Enfermería, Úlcera por presión, Urgencias médicas, Personal de salud.

\section{INTRODUÇÃO}

As lesões por pressão (LPP) são consideradas eventos adversos de grande incidência, as quais agravam o quadro clínico do paciente, geram infecções graves, ocasionam aumento de tempo de internação, elevam os custos e acrescem os casos de depressão em pacientes (SOUSA RC e FAUSTINO AM, 2019. A Agência Nacional de Vigilância Sanitária apresenta, em seu relatório os números de casos notificados: 19.297, sendo que destes, 6.101 são lesões de estágio I, 10.438 são lesões de estágio II, 1.720 são lesões de estágio III, 520 são lesões de estágio IV e 518 são lesões sem informação (ANVISA, 2018).

Diante deste contexto, a ANVISA e o Ministério da Saúde lançam o Programa Nacional de Segurança do Paciente, com o foco nos problemas de maior incidência, criado pela portaria 529/2013, com o objetivo diminuir a incidência dos eventos adversos no cuidado. Esta portaria está em acordo com protocolos internacionais que, entre suas metas, estabelecem a redução do risco de LPP (BRASIL, 2013).

A prática do cuidado de pacientes com lesões cutâneas é uma especialidade dentro da área de enfermagem, reconhecida pela Sociedade Brasileira de Enfermagem Dermatológica (SOBEND) e pela Associação Brasileira de Estomaterapia (SOBEST), dando ao enfermeiro respaldo técnico-científico para exercer sua autonomia no cuidado destas lesões (SOUZA EM, et al., 2013).

Desta forma, entende-se que o conhecimento relacionado ao desenvolvimento de LPP é essencial para o enfermeiro, pois o mesmo é responsável por direcionar as intervenções que possibilitem a criação e implementação de estratégias de prevenção, avaliação e tratamento de LPP. Entretanto, estudo realizado com 95 enfermeiros, em um hospital de ensino do interior de Minas Gerais, apontou algumas fragilidades no conhecimento dos profissionais, ao utilizarem técnicas de prevenção ultrapassadas, o que demonstrou a necessidade de atualização e capacitação em serviço (CAMPO IALM, et al., 2019).

Nesse sentido, torna-se necessário identificar o conhecimento dos profissionais acerca dos aspectos relativos as LPPs. Dessa forma este estudo justifica-se pela necessidade de identificação de possíveis lacunas na habilidade e competência sobre o manejo de LPP, de modo a subsidiar a implementação de medidas educativas eficientes, sanar as deficiências e melhorar a qualidade do cuidado ofertado no Pronto Atendimento referente ao cuidado prestado às pessoas com LPP. Frente ao exposto, questiona-se: Qual o conhecimento de enfermeiros atuantes em um serviço de urgência sobre as lesões por pressão? Na tentativa de responder o questionamento, objetivou-se verificar o conhecimento dos enfermeiros atuantes em um pronto atendimento sobre lesão por pressão.

\section{MÉTODOS}

Trata-se de uma pesquisa exploratória, descritiva de abordagem qualitativa, desenvolvida com enfermeiros atuantes em um Pronto Atendimento Municipal (PAM), com atendimento SUS, localizado no Rio Grande do Sul (RS), Brasil.

O cenário do estudo realiza atendimento 24 horas/dia, é referência para atendimentos clínicos adultos e pediátricos os quais são divididos dentro da mesma estrutura física. Recebe pacientes de livre demanda caracterizados como baixa e média complexidade, encaminhados da Atenção Básica; trazidos pelo Serviço de Atendimento Móvel de Urgência (SAMU); Bombeiros e outros serviços pré-hospitalares para estabilização, manejo e encaminhamentos que se fizerem necessários. 
O serviço contava no momento da pesquisa com 14 enfermeiros, com escala de trabalho de 12/36 horas, que atenderam aos critérios de inclusão: ser enfermeiro, com atuação mínima de seis meses no serviço, que atuavam no atendimento à pacientes adultos. Atenderam aos critérios, formando o Corpus dessa pesquisa, 12 enfermeiros. Os profissionais foram convidados a participar da pesquisa, pessoalmente e de forma individual, durante o plantão no mês novembro de 2020. Após o aceite dos mesmos, um dos pesquisadores, e procedeu a coleta dos dados, através de um questionário contendo questões mistas, norteadas pela técnica vinheta (GALANTE AC, et al, 2003).

A vinheta é uma ferramenta utilizada para a coleta de dados, onde os participantes são convidados à compartilhar, se posicionar e comentar sobre breves descrições, com situações específicas, fictícias ou reais. Por meio dela, se identifica a conduta do pesquisado em determinada situação, o que contribui como ponto de partida para resolução de problemas. Para tanto, os participantes foram convidados a refletirem sobre a seguinte situação hipotética e analisar a representação de uma lesão por pressão: Usuária, WXS, gênero feminina, 68 anos de idade, 1,63 de altura, 53kg, acamada. Internada a 2 meses. Patologias prévias: HAS, DM, AVC, tabagista à 50 anos. Desorientada, em permanência de leito, com pouca sensibilidade, dieta via sonda nasoentérica, eliminações fisiológicas em fralda a nove dias surgiu uma lesão por pressão (LPP), localizada no cóccix.

Após, foram instruídos a responder aos seguintes questionamentos: Na sua opinião, quais fatores contribuem para o visualizado na figura? Qual o estadiamento e os tecidos presentes na lesão apresentada? Qual o escore de Braden você atribui a lesão da figura. Realize uma prescrição de enfermagem para essa situação. Você visualiza potencialidade/fragilidades no seu cotidiano profissional no que se fere ao cuidado/tratamento de pessoas com LPP? Quais estratégias podem ser pensadas nesse contexto?

A análise dos dados, ocorreu com base na técnica da análise de conteúdo, de Minayo MC (2014), organizada a partir de uma sequência de três componentes: pré-análise, exploração do material e tratamento e interpretação dos resultados. Na primeira etapa, os pesquisadores realizaram a leitura flutuante do material com objetivo de apropriar-se do seu conteúdo; na segunda, realizou-se inicialmente a codificação dos relatos, o que resultou em uma unidade de registro, a partir da identificação das vivenciadas dos enfermeiros no tratamento/cuidado de pessoas com lesão por pressão. A mesma foi dividida em três unidades de contexto; na primeira unidade agrupou-se as questões relativas ao conhecimento dos enfermeiros sobre a lesão por pressão; na segunda, as fragilidades vivenciadas pelos enfermeiros no contexto de cuidados a pessoas com lesão por pressão; e na terceira, as estratégias sugeridas pelos enfermeiros para auxiliar na superação de algumas fragilidades.

Após, foi realizada nova leitura a partir da unidade de registro das de contexto, momento em que cada relato inserido nas unidades de base foi lido de forma minuciosa, sendo separados em diferentes nas unidades, dando origem as categorias. Por fim, procedeu-se a última etapa do método de análise, onde o pesquisador apresentou a interpretação dos resultados obtidos.

Foram considerados os preceitos éticos que envolvem a pesquisa com seres humanos, conforme a Resolução 466/2012 e a resolução 510/2016 do ministério da saúde. (BRASIL, 2012; BRASIL, 2016). O Projeto foi aprovado por Comitê de Ética em Pesquisa pelo Parecer número: 4.390.762e CAAE:38800120.60000.5306. Os participantes foram identificados pelas letras $E$ (enfermeiro) seguidas de um algarismo numérico (E1, E2...E12).

\section{RESULTADOS}

Dos 12 participantes, dois eram do sexo masculino e 10 do feminino, com idades entre 32 a 59 anos e tempo de formação entre sete e 34 anos. Destes, 11 possuíam título de especialista e 1 era generalista. Todos possuíam regime de trabalho estatutário e escala de 12/36, divididos em turno diurno e noturno.

As respostas relativas as questões fechadas do instrumento versaram sobre os tecidos apresentados na figura e sobre a escore atribuídos pelos profissionais utilizando a escala de Braden, de forma que sete profissionais apontaram grau 2, dois apontaram grau 3, dois apontaram grau 1 e um não soube responder. Em relação aos tecidos presentes na lesão foram citados: Derme e epiderme (E1, E2, E4, E5, E6, E7, E9, 
$E 11, E 12)$, tecido de granulação (E3, E10); tecido muscular e epitelias (E8, E11); profundo, adiposo, muscular e osso (E12). Quanto ao escore baseando na escala de Braden, um profissional avaliou como escore 07 (E1); um como o escore 08 (E10), um como escore 12 (E8).; três avaliaram com o escore 09 (E5, E9, E12) e seis com escore 11 (E2, E3, E4, E6, E7, E11). Os dados das questões abertas resultaram em uma unidade de registro: Vivências de enfermeiros no tratamento/cuidado as pessoas com lesão por pressão. A mesma foi unitarizada em três unidades de contexto e cinco categorias, conforme pode ser visualizado na (Figura 1).

Figura 1 - Representação esquemática da integração entre a unidade de registro, unidades de contexto e categorias.

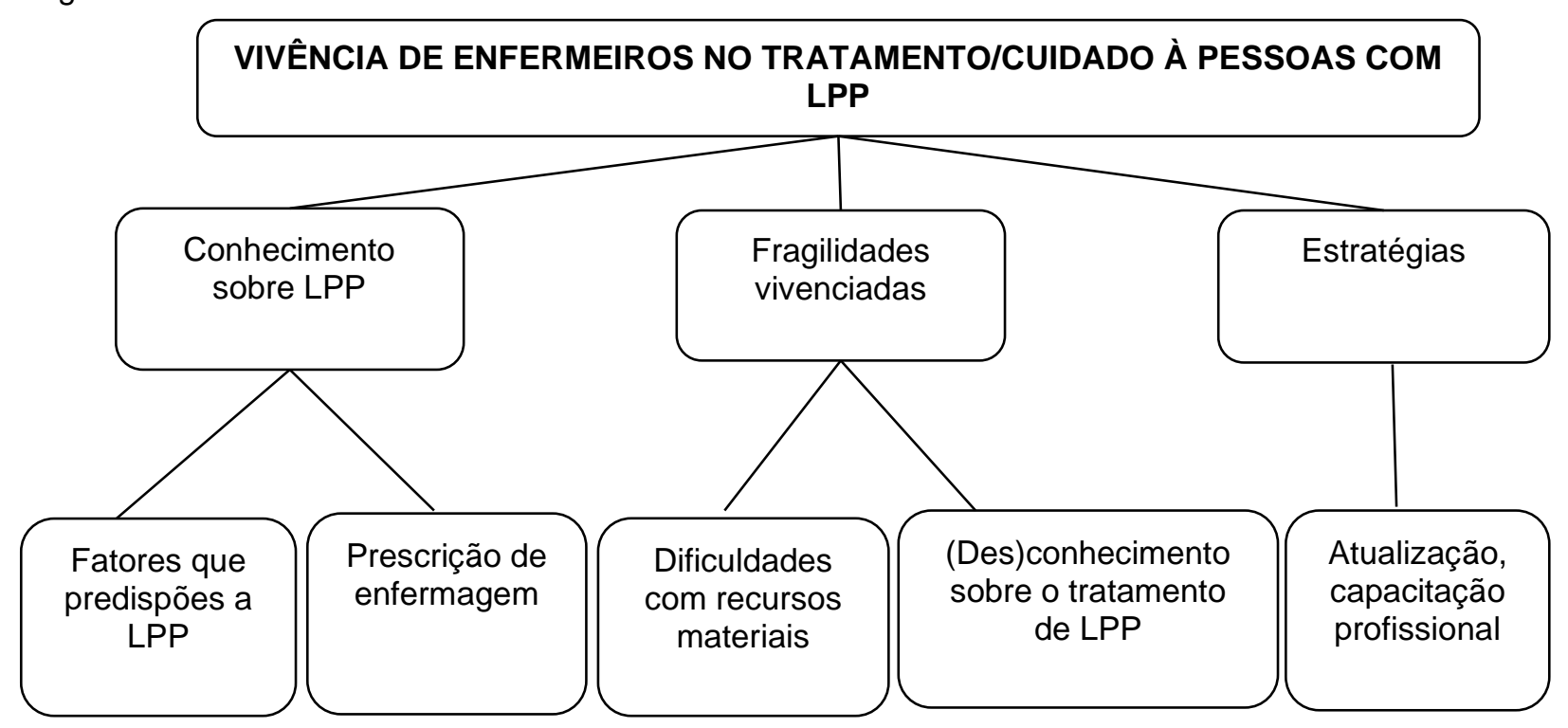

Fonte: Panerai GSS, et al., 2021.

\section{Fatores que predispões a lesão por pressão: percepção dos enfermeiros}

Para realizar a assistência com qualidade e de forma integral deve-se ter em mente os elementos que podem desencadear a ocorrência de LPP, dependendo da identificação dos profissionais sobre os diversos fatores que interagem entre si, dentre os quais estão os relacionados aos pacientes e à própria instituição, como provedora do cuidado. Em relação aos dados coletados observou-se que os profissionais possuem conhecimento significativo acerca das condições que levam à LPP, entre os fatores citados estão a limitação da mobilidade, alteração no nível de consciência, incontinência urinária, diabetes mellitus e tabagismo.

"Eliminações fisiológicas em fralda, dieta por sonda, diabética" (E1).

"Falta de alteração de decúbito; pouca hidratação do local" (E4).

"Permanência no leito, imobilidade; patologias prévias como Diabetes Mellitus, o Acidente Vascular Cerebral prévio; fraldas; nutrição por Sonda Nasogástrica (se não for a indicada); idade; desorientação e pouca sensibilidade, local da pressão; local da lesão" (E5).

"Tempo de internação, idade, falha na mudança de decúbito" (E7).

"Patologias como Diabetes Mellitus, sequela de Acidente Vascular Cerebral prévio, tabagista, acamada (restrita ao leito), perda de sensibilidade" (E10).

\section{Prescrição de enfermagem para pessoas com lesão por pressão}

O enfermeiro é parte integrante da equipe multiprofissional de saúde, responsável pela tomada de decisão que propicia a escolha da melhor prática do cuidado a ser empregada ao paciente na busca da qualidade da assistência. Dentre as atribuições do profissional enfermeiro está a realização de um exame físico detalhado, 
avaliação e caracterização das lesões apresentadas pelo paciente e prescrição dos cuidados. Em relação aos cuidados que os pacientes necessitam apresentou-se a troca de decúbito, curativo, alimentação, hidratação.

"Solicitação de sondagem vesical prescrito pelo médico; mudança de decúbito de duas em duas horas, curativo, colchão piramidal, massagem de conforto" (E1).

"Verificar sinais vitais; mudança de decúbito de duas em duas horas, higienização no leito uma vez ao dia; curativo duas vezes ao dia; dieta por sonda nasoentérica, cuidados com alimentação" (E2).

"Mudança de decúbito de duas em duas horas; curativo duas vezes ao dia ou conforme necessidade; hidratação no intervalo da infusão da dieta, controle da glicemia; uso de colchão piramidal ou pneumático" (E3).

Em contraponto alguns profissionais descreveram um pouco diferente os cuidados, como a orientação em relação ao número de curativos realizados diariamente e o tempo entre a mudança de decúbito.

"Verificar a dieta via sonda nasoentérica, se adequada à paciente conforme patologias prévias e condições clínicas atuais. Orientar troca de fraldas seguida, orientar alternância de decúbito e massagens de conforto em proeminências ósseas, proteger com almofadas/coxins, outras alternativas que aliviem a pressão, orientar a troca de roupa de cama, sempre que úmida ou necessidade e esticar bem para evitar pregas" (E5).

"Mudança de decúbito de duas em duas horas; curativo três vezes ao dia" (E8).

"Realizar curativo na lesão uma vez ao dia; realizar alternância de decúbito da paciente; instalar dieta por sonda nasoentérica conforme prescrição” (E10).

"Mudança de decúbito de três em três horas, uso de emolientes na pele, colchão piramidal para diminuição do atrito com a pele nas proeminências ósseas" (E12).

\section{Dificuldades com recursos materiais para tratamento/cuidado de pessoas com LPP}

Outro aspecto que apresenta problemas frequentes diz respeito a disponibilidade de materiais suficientes para o cuidado com as lesões. Apesar de poder contar com materiais básicos, ocorre a falta ou ausência de materiais específicos. Os profissionais contam com auxílio de um centro de referência na cidade.

"Materiais como gaze, solução fisiológica, micropore não faltam; curativos de cobertura não tem o suficiente" (E1).

"Não, falta muita cobertura que poderíamos usar, Os nossos curativos, devido a falta de curativos de cobertura, são simples, geralmente com solução fisiológico e gaze para cobertura" (E7).

"Não quando precisamos recorremos á outro serviço" (E8).

“Em certos períodos sim, não o suficiente, porém há materiais disponíveis" (E9).

"Não por se tratar de um serviço de pronto atendimento e não como unidade de internação" (E10).

"O serviço disponibiliza um pouco de materiais como as coberturas, mas o posto é referência para este tipo, quando possível encaminha-se, aqui, utiliza-se ainda solução fisiológica e gases" (E12).

\section{(Des)conhecimento sobre o tratamento/cuidado com pessoas com LPP}

Os participantes da pesquisa responderam que não se sentem capacitados para realização do cuidado aos pacientes portadores de LPP, e também referem a necessidade de atualização e capacitação para lidar com esta comorbidade, considerando as novas coberturas para a realização de curativos em que 0 profissional enfermeiro precisa ter conhecimento para prestar uma assistência de qualidade. 
"Não me sinto capacitada para curativos de cobertura, falta continuidade do tratamento, sim para curativos básicos" (E2).

"Não me sinto capacitada, tenho pouca vivência nessa área e preciso me capacitar mais" (E4).

"Não me sinto capacitada, pois não tenho capacitação específica atualizada" (E6).

"[...] No momento me sinto pouco capacitada" (E8).

"[...] Realizamos tal procedimento sem capacitação, com materiais básicos [...]" (E11).

\section{Atualização/capacitação profissional para tratamento/cuidado de pessoas com LPP}

O estudo acerca de LPP, demonstrou que a vivência profissional impõe desafios diários. O contato frequente com o paciente expõe problemas e fragilidade pessoais e do sistema. Observou-se a necessidade de constante aprimoramento profissional e como a presença ou ausência do mesmo impacta diretamente nas tomadas de decisão sobre os cuidados com paciente, conforme identifica-se nas falas abaixo:

\section{"[...] Capacitação quanto ao uso de curativos modernos faz falta" (E1).}

"[...] Há necessidade de aprofundar os conhecimentos e conhecer materiais específicos bem como ter acesso aos mesmos para uso nas lesões" (E3).

"Acredito na importância das atualizações e capacitações [...]" (E8).

"[...] É necessários curso de reciclagem e capacitação com mais frequência” (E9).

\section{DISCUSSÃo}

A vivência em um pronto atendimento, impõe diversos desafios aos profissionais da saúde, dentre eles o cuidado as pessoas que convivem com LPP, a qual é caracteriza como um dano localizado na pele e/ou tecidos moles subjacentes, geralmente sobre proeminência óssea ou ainda relacionada ao uso de equipamento medico ou outro objeto (NPUAP, 2016). Nos serviços de atendimento em urgência, a avaliação e estabilização dos pacientes acontece na sala de emergência, local onde estão os usuários com risco elevado de LPP. Neste sentido, a avaliação do paciente na chegada é essencial para o plano de cuidado e desfecho benéfico (SILVA DP, et al, 2020). No pronto atendimento a LPP pode se desenvolver rapidamente como consequência do uso destes equipamentos, razão pela qual devem ser retirados assim que possível (ATLS, 2012).

Na presente pesquisa, verificou-se que os profissionais possuíam conhecimento significativo acerca das condições que levam à formação de LPP. Dentre os fatores citados, destacaram-se a limitação da mobilidade, alteração no nível de consciência, incontinência urinária, diabetes mellitus e tabagismo. Esse dado, assemelha-se ao encontrado em um estudo realizados com enfermeiros que avaliou 33 itens relativos à prevenção de LPP, sendo que em 17 itens obteve-se 90 a 100\% de acertos e em cinco itens 70 a 89,9\% de acertos. Contudo, seis itens apresentaram resultado menor que $50 \%$, demonstrando que a equipe possui um déficit de conhecimento sobre o assunto (BONFADA MS, et al, 2018).

No que diz respeito aos cuidados que os pacientes necessitam com vistas a prevenção de LPP, alguns participantes da presente pesquisa referiram a alternância de decúbito, curativo, alimentação, hidratação. Em contraponto, outros profissionais descrevem um pouco diferente os cuidados, como a orientação em relação ao número de curativos realizados diariamente e o tempo entre a mudança de decúbito. Estudo desenvolvido com revisão integrativa de literatura demonstra que alguns fatores contribuem para o sucesso na prevenção de LPP, tais como o conhecimento e competência dos profissionais de saúde sobre o assunto, cuidado integral e individualizado, metas determinadas, emprego de escalas preditivas, procedimentos para alívio da pressão e elaboração de medidas de prevenção e tratamento de LPP (FREIRE DA, et al., 2020).

Com o intuito de qualificar o cuidado, monitorar a incidência e constituir planejamento institucional para minimizar estes agravos, foi criado o Programa Nacional de Segurança do Paciente, visto que o desenvolvimento das práticas preventivas durante a internação hospitalar é um importante indicador da qualidade da assistência (BRASIL, 2013). 
Quanto maior o conhecimento do enfermeiro, maior a probabilidade de reconhecer os fatores que levam à formação das LPP, e possibilita a realização de uma prescrição de enfermagem adequada que considere o controle da incontinência urinária pois a diurese em contato com a pele causa irritação química, maceração por umidade, dermatite, colonização de bactérias e fungos, comprometendo a integridade; a troca de decúbito a cada duas horas, em uso de cadeira de rodas a cada uma hora, com o objetivo de evitar que a pressão exercida sobre o leito, associada à fricção e cisalhamento comprometam o fluxo sanguíneo e causem rompimento da pele; a utilização de redutores de pressão, mobilização adequada no leito e proteção das proeminências (OLIVEIRA DMN, et al., 2019).

Observar a limpeza e umidade da pele e a aplicação de cremes hidratantes à base de ácidos graxos que servem de barreira contra a umidade, também devem ser práticas constantes para prevenção e/ou tratamento de LPP. Também deve-se prestar especial atenção à hidratação e nutrição do paciente, sendo a avaliação nutricional uma importante prática, pois, a ferida pode provocar inúmeros efeitos infeciosos no organismo, os quais aumentam a necessidade nutricional dificultando o processo de reparação e reconstrução do tecido, decorrentes da cicatrização. Destaca-se a necessidade de avaliação do enfermeiro para a escolha de materiais e dispositivos que possibilitem um maior benefício ao paciente que apresenta LPP. A dor é subjetiva de cada um, e sua avaliação é primordial (OLIVEIRA DMN, et al., 2019).

Dentre as fragilidades vivenciadas pelos enfermeiros participantes da presente pesquisa, está a indisponibilidade de materiais suficientes para o cuidado com as lesões. Apesar de poder contar com materiais básicos, ocorre a falta ou ausência de materiais específicos. Em uma pesquisa com o objetivo de descrever as recomendações sobre os recursos humanos e materiais direcionados para a prevenção de LPP, foram apresentados os recursos disponíveis no mercado, mas não se identificou os materiais disponíveis em unidades hospitalares (TONOLE R e BRANDÃO ES, 2018). Salienta-se que cabe ao enfermeiro avaliar quais materiais e dispositivos devem ser usados, sendo necessário suporte institucional para a implementação de práticas seguras e fornecimento de recursos materiais e humanos que corroborem as boas práticas e proponham e implementem protocolos de prevenção (BRASIL, 2017). Contudo, os enfermeiros devem estar atualizados nos tratamentos inovadores e terapêuticos e suas coberturas com diversas tecnologias (CAUDURO FP, et al., 2018; SOUZA E, et al., 2020).

A ausência de capacitação, também foi mencionada, na presente pesquisa como uma fragilidade vivenciada pelos enfermeiros. Em uma pesquisa realizada em um hospital público de médio porte, concluiuse que $90 \%$ da equipe possuía conhecimento sobre formas de prevenção a LPP, embora o mesmo número obtivesse média abaixo do esperado no quesito classificação da LPP. O estudo apontou algumas fragilidades no conhecimento, considerando que muitos profissionais ainda utilizam técnicas ultrapassadas na prevenção e tratamento de LPP (JUNIOR LJS, et al., 2019).

Como estratégia para auxiliar na superação das dificuldades vivenciadas, os participantes da presente pesquisa salientaram a necessidade de atualização e capacitação para lidar com as pessoas com LPP, considerando as novas coberturas para a realização de curativos que o enfermeiro precisa conhecer para desenvolver um cuidado de qualidade. Faz parte das atribuições das instituições priorizar a elaboração e implementação de protocolos de prevenção para melhoria da qualidade assistencial. Preparar permanentemente o profissional no tocante ao tema prevenção possibilita um enfoque baseado no paciente, em suas necessidades e gestão de cuidado baseado em evidências científicas (MENDONÇA PK, et al., 2018).

O uso de protocolos atribui maior autonomia, organização e sistematização da assistência as pessoas com LPP, respaldando as condutas de avaliação, diagnóstico, planejamento, cuidado, tratamento e evolução da ferida (CHIBANTE CLP, et al., 2015). Compreende-se dessa forma, que os instrumentos construídos com base científica, podem com a atividade do enfermeiro. Assim, é fundamental que as instituições construam seus protocolos, ampliando a atuação do enfermeiro, conforme o que é permitido pelo Conselho Federal de Enfermagem (COFEN) (CARDOSO DS, et al., 2019).

A Política Nacional de Educação Permanente (EPS) contribui às estratégias de organização do exercício da atenção, da gestão, da formação construída na prática das equipes (BRASIL, 2017; BRASIL 2009). A EPS traz vários benefícios, permitindo em curto prazo proporcionar condições de melhorias do desempenho 
técnico dos enfermeiros, prevenindo falhas e valorizando a ciência como fonte de conhecimento. A médio e a longo prazo, pode favorecer a reflexão crítica do trabalho, articulando o arcabouço teórico com a prática profissional (BRASIL, 2009; MONTANHA D e PEDUZZI M, 2016).

\section{CONCLUSÃO}

Essa pesquisa, pois possibilitou verificar o conhecimento dos enfermeiros atuantes em um pronto atendimento sobre lesão por pressão. Como principais resultados, evidenciou-se que os profissionais enfermeiros são fundamentais para realizar o cuidado de pacientes com LPP, demonstrou que possuem conhecimento sobre os fatores que predispõe, e realizam plano de cuidados necessários; observou-se também, que os profissionais não se sentem capacitados e que sentem necessidade de realizar treinamentos, capacitações para atualização. Salienta-se que ao considerar as unidades de pronto atendimento como porta de entrada para os usuários do sistema de saúde, conhecer a realidade do serviço possibilita que protocolos sejam implantados para sustentar a pratica e gerar benefícios na qualidade da assistência em enfermagem.

\section{REFERÊNCIAS}

1. AGÊNCIA NACIONAL DE VIGILÂNCIA SANITÁRIA (ANVISA). Boletim Segurança do Paciente e Qualidade em Serviços de Saúde no 20. 2018 [publicado em 2020]. Disponível em:https://www.gov.br/anvisa/ptbr/centraisdeconteudo/publicacoes/servicosdesaude/boletimseguranca-do-paciente. Acesso: 26 janeiro de 2021.

2. ATLS. Advanced Trauma Life Support for Doctors. American College of Surgeons. 9a . Ed, 2012.

3. BONFADA MS, et al. Potencialidades e limites da autonomia do enfermeiro em ambiente hospitalar. Rev enferm UFPE online., 2018; 12(8): 2235-46.

4. BRASIL. Política Nacional de Educação Permanente em Saúde. Brasília: Ministério da Saúde, 2009.

5. BRASIL. Ministério da saúde. Agência nacional de vigilância sanitária. Nota técnica GVIMS/GGTES № 3/2017: praticas seguras para prevenção de lesão por pressão em serviços de saúde. Brasilia: Ministério da Saúde; 2017.

6. BRASIL. Portaria no 529, Institui Programa Nacional de Segurança do Paciente. Brasília: Ministério da Saúde, 2013.

7. BRASIL. Resolução n.466, de dezembro de 2012: Aprova as diretrizes e normas regulamentadoras de pesquisa envolvendo seres humanos. Brasília (DF); 2013.

8. BRASIL. Resolução № 510 , de 07 de abril de 2016, Art.1ํㅜㄹ item VII. Dispõe sobre as normas aplicáveis e pesquisas em Ciências Humanas e Sociais. Diário Oficial da União. 2016, 07 de abril.

9. CAMPO IALM, et al. Educação permanente para boas práticas na prevenção de lesão por pressão: quaseexperimento. Rev Bras Enferm. 2019; 72(6): 1725-31.

10. CARDOSO DS, et al. Conhecimento dos Enfermeiros sobre Classificação e Prevenção de Lesão por Pressão. Rev Fund Care Online, 2019; 11(3): 560-566.

11. CAUDURO FP, et al. Atuação dos enfermeiros no cuidado das lesões de pele. Rev enferm UFPE online., 2018; 12(10): 2628-34.

12. CHIBANTE CLP, et al. Perfil de clientes hospitalizados com lesões cutâneas. Rev. cuba. Enferm, 2015; 31(4).

13. FREIRE DA, et al. Variáveis associadas à prevenção das lesões por pressão: conhecimento para o cuidado de enfermagem. R. pesq: cuid. fundam. Online, 2020; 12: 1172-1178.

14. GALANTE AC, et al. A vinheta como estratégia de coleta de dados de pesquisa em enfermagem. Rev Latino-am Enfermagem, 2003; 11(3): 357-63.

15. JUNIOR LJS, et al. Educação Permanente; Ferramenta de aprimoramento assistencial ás lessões por pressão. Rev enferm UFEPE online, 2019; 13(5): 1115-23.

16. MENDONÇA PK, et al .ocorrência e fatores de risco para lesões por pressão em centros de terapia intensiva. Rev enferm UFPE online, 2018; 12(2): 303-11.

17. MINAYO MC. O desafio do conhecimento: pesquisa qualitativa em saúde. 11. ed. São Paulo, SP: Hucitec, 2014.

18. MONTANHA D, PEDUZZI M. Permanent education in nursing: survey to identify the necessities and the expected results based on the workers conception. Rev Esc Enferm USP, 2016; 44(3): 597-604.

19. NATIONAL PRESSURE ULCER ADVISORY PANEL (NPUAP). NPUAP announces a change in terminology from pressure ulcer to pressure injury and updates the stages of pressure injury [Internet]. 2016.

20. OLIVEIRA DMN, et al. Intervenções de enfermagem para pacientes com lesão por pressão. Rev enferm UFPE online, 2019, 13: e 240237.

21. SILVA DP, et al. Risco de lesão por pressão entre usuários de unidades de pronto atendimento. Rev Gaúcha Enferm., 2020; 41: e20190334.

22. SOUSA RC, FAUSTINO AM. Conhecimento de enfermeiros sobre prevenção e cuidados de lesão por pressão. Res.: fundam. care. Online, 2019; 11(4): 992-997.

23. SOUZA E, et al. Avaliação e tratamento de lesões por pressão na estratégia saúde da família. Rev enferm UFPE online, 2020; 14: e243522.

24. SOUZA EM, et al. Ulcer dueto chronic venous disease: a socio demographic study in North eastern Brazil. Ann Vasc Surg., 2013; 27(5): 571-6.

25. TONOLE R, BRANDÃO ES. Recursos humanos e materiais para a prevenção de lesão por pressão. Rev enferm UFPE online, 2018; 12(8): 2170-80. 